\title{
Realization of Serbian accents by Serbian and Russian speakers
}

Ekaterina Panova

Department of history and theory of language, St. Tikhon's Orthodox University, Russia

https://doi.org/10.36505/ExLing-2015/06/0015/000252

\begin{abstract}
The present study examined realization of Serbian pitch accents by two Serbian and four Russian female speakers in initial, medial and final position of the statements. For each word the set of pitch parameters were calculated. The main statistical differences between realizations of rising and falling accents by Serbian speakers were found with respect to F0 start value and timing of F0 maximum. These pitch parameters also served as an effective indicator of deviations which were observed across Russian speakers. The main difficulties for Russian speakers were concerned realizations of falling accents in initial and medial positions.
\end{abstract}

Key words: pitch contour, pitch accent, Russian, Serbian.

\section{Introduction}

One of the main differences between Russian and Serbian stress is a tone character of the latter. Traditionally, it has been established that standard Serbian has four accents, which differ in pitch (rising or falling) and duration (short or long): long rising (LR), long falling (LF), short rising (SR) and short falling (SF). The problem of the distinction between falling and rising accents is discussed in many investigations (for example, in Leniste 1986, Keijsper 1987). The most valuable parameters of the distinction are range of F0 between accented and post-tonic syllable and location of the F0 peak.

Russian stress is mostly characterized by duration and vowel quality. In Russian F0 is the main parameter of intonation, since Russian belongs to the languages with strong influence of intonation on word prosody (Nikolaeva 1977). In Serbian intonation also has a great influence on word prosody, although types of accents can modify its contour. In final position of the statements there is a tendency in neutralization of the accents (Lehiste et al. 1986).

Despite the fact that within the last decade many phonetic studies of Serbian and Russian have appeared, there are no comparisons of prosodic features between these languages except one extensive study, which was conducted in 1970's by Nikolaeva (Nikolaeva 1977). The work presented here forms part of a larger study which aims to investigate how Russian speakers realize Serbian accents and develop the methodology for training Serbian accents by Russian speakers.

ExLing 2015: Proceedings of 6th Tutorial and Research Workshop on Experimental Linguistics, 26-27 June 2015, Athens, Greece 


\section{Method}

For the present study 111 target words of mono-, di- and tri-syllables with different vowel inventory and different types of accent were selected from available dictionaries. For minimizing segmental influences only words with unvoiced consonants were selected. Each target word was embedded in frame sentences so as to occur in initial, medial and final position. All the sentences were statements.

Then, two control native speakers of Serbian (S1, S2, females) and four Russian speakers (R1, R2, R3, R4, females) read these sentences in neutral style and normal tempo.

For both Serbian and Russian sentences the contour of F0 was calculated. Visual and auditory analyses were conducted to eliminate all misreadings and errors in calculation of F0. From contours of the target words the set of pitch parameters (described in detail in (Smirnova et al. 2007) were obtained and then processed with the STATISTICA software program.

\section{Results}

Here the results for two pitch parameters, F0 start value and timing of F0 maximum are presented.

\section{F0 start value}

A two-way ANOVA with repeated measures (type of accent $\mathrm{x}$ position) was performed for Serbian and Russian speakers separately. For this statistical analysis four types of Serbian accents were divided only in two types: falling (FA) and rising (RA).

Post hoc tests Tukey HSD indicated that there was a significant difference between falling and rising accents for Serbian speakers in initial $(\mathrm{p}<.0001)$ and medial positions $(\mathrm{p}<0.0001)$ and only for one Serbian speaker $(\mathrm{S} 1 \mathrm{p}=0.226, \mathrm{~S} 2$ $\mathrm{p}<0.0001)$ in final position. F0 start value serves as an indicator of the types of accents: rising accents have lower F0 start value, than falling ones. This difference between accents can be observed even in final position (i.e. nucleus), which usually influences much on word prosody.

Across Russian speakers there was no significant difference between the types of accents $(p=1.000)$ except for one Russian speaker, who has a significant difference only in medial position $(R 3 \mathrm{p}=0.001)$. Although some Russian speakers may have the same tendency as Serbian speakers, they don't demonstrate any significant dependence (Figure 1). 

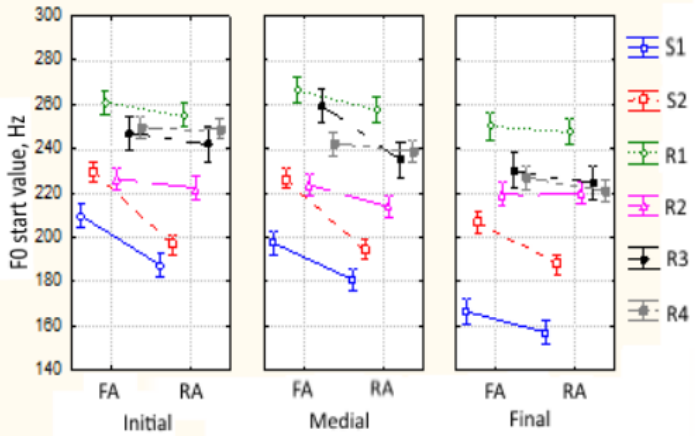

Figure 1. F0 start value scores of the words with falling (FA) and rising accents (RA) for Serbian (S1, S2) and Russian speakers (R1, R2, R3, R4) in initial, medial and final position.

\section{Timing of F0 maximum}

For the estimation of timing of F0 maximum the Survival Analysis was performed with type of accent as the grouping variable. For Serbian speakers there was a significant difference between accents in initial and medial position $(p<0.0001)$, but not in final position $(p>0.1)$. Thus, falling accents mostly have earlier peak locations (up to $40 \%$ ) and rising accent have later peak locations (from 50\%). In final position the distinction between rising and falling accents is neutralized (Figure 2).
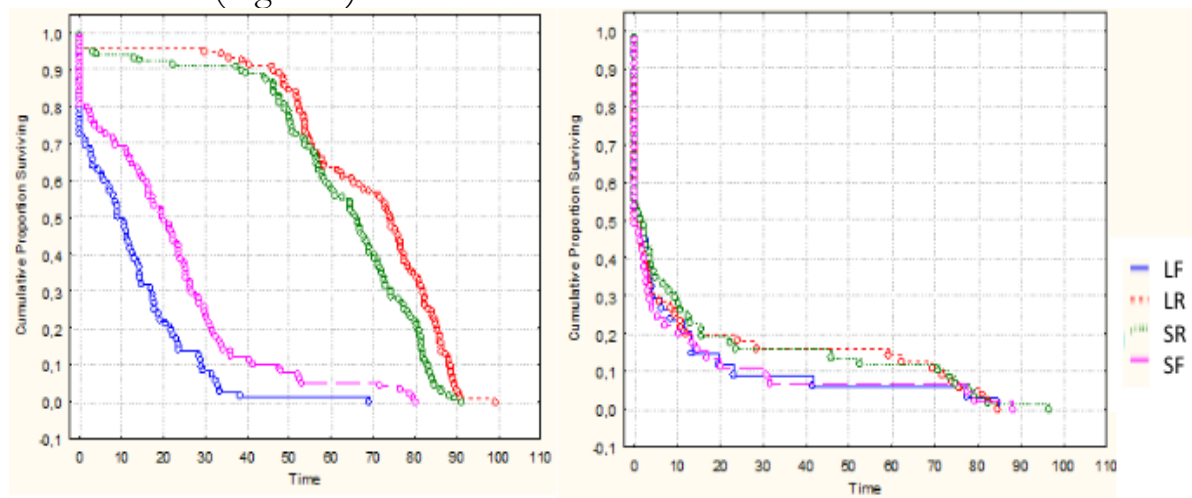

Figure 2. Timing of F0 maximum scores of words with falling (FA) and rising accents (RA) in initial, medial (left) and final position (right) for Serbian speakers.

Russian speakers didn't have strong tendency between the types of accents in timing of F0 maximum in all positions ( $\mathrm{p}>0.1)$. The values of timing of F0 
maximum showed that Russian speakers mostly realized "rising" accents instead of falling ones (from $50 \%$, Figure 3).
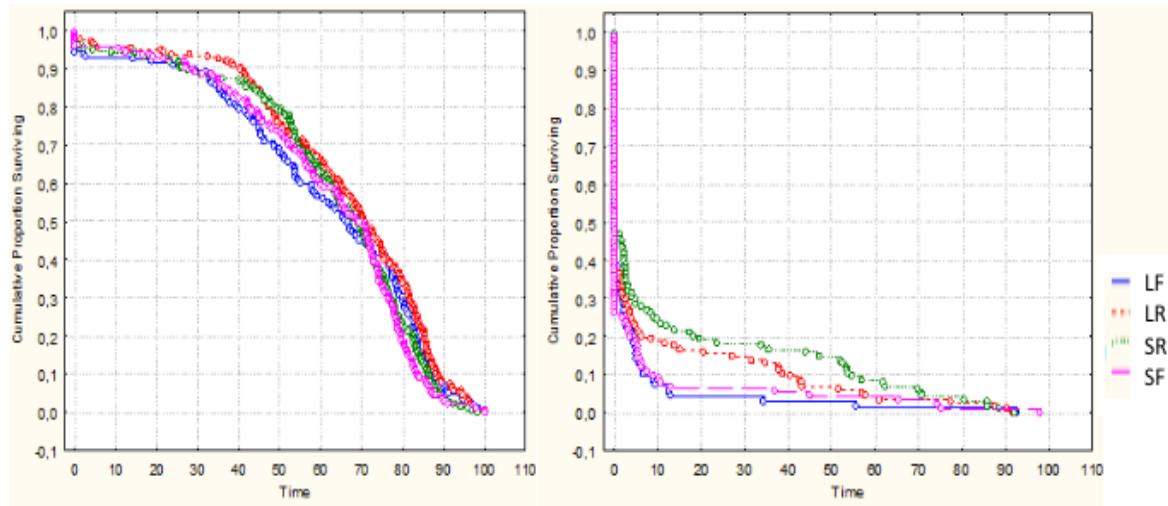

Figure 3. Timing of F0 maximum scores of words with falling (LF, SF) and rising accents (LR, SR) in initial, medial (left) and final position (right) for Russian speakers.

\section{Conclusion}

The results of the present study showed that pitch parameters, F0 star value and timing of F0 maximum, allowed to provide the distinction between Serbian rising and falling accents, and served as an effective indicator of deviations which were observed across Russian speakers.

Although previous researches of Serbian accentuation mentioned parameters of F0 star value (Lehiste 1981), it isn't interpreted as a one of the most distinctive between rising and falling accents.

The main difficulties in realizations of Serbian accents by Russian speakers are related to falling accents in initial and medial position. In final position the neutralization of the accents is observed across both Serbian and Russian speakers.

\section{References}

Keijsper, C.E 1987. Studing Neoštokavian Serbocroation Prosody. Dutch Studies in South Slavic and Balkan Linguistics. SSGI 10, 101-193.

Lehiste I., Ivic P. 1986. Word and sentence prosody in Serbocroatian. Cambridge, Mass., MIT Press.

Nikolaeva T.M. 1977. Frazovaja intonacija slavianskih jazykov. Moskva, Nauka.

Smirnova, N., Starshinov A., Oparin I. \& Goloshchapova T. 2007. Speaker Identification Using selective Comparision of Pitch Contour Parameters. Proc. of the $16^{\text {th }}$ ICPhS, Saarbrucken, 203-206. 